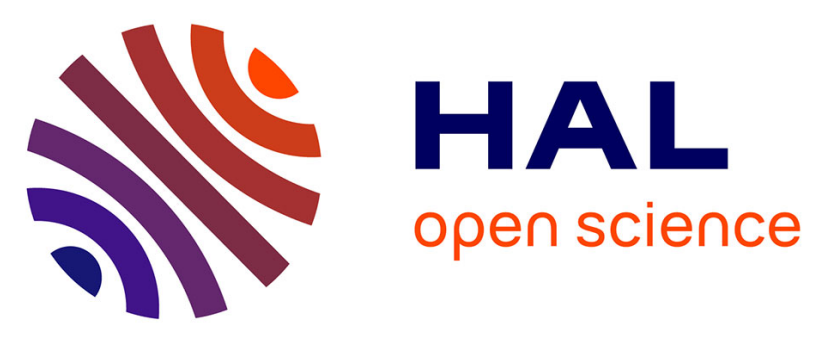

\title{
On the occurrence of size effects in the calculation of thermal conductivity by first-principles molecular dynamics: The case of glassy GeTe4
}

Évelyne Martin, Pier Luca Palla, Fabrizio Cleri, Assil Bouzid, Guido Ori, Sébastien Le Roux, Mauro Boero, Carlo Massobrio

\section{To cite this version:}

Évelyne Martin, Pier Luca Palla, Fabrizio Cleri, Assil Bouzid, Guido Ori, et al.. On the occurrence of size effects in the calculation of thermal conductivity by first-principles molecular dynamics: The case of glassy GeTe4. Journal of Non-Crystalline Solids, 2018, 498, pp.190-193. 10.1016/j.jnoncrysol.2018.05.014 . hal-02128447

\section{HAL Id: hal-02128447 \\ https://hal.science/hal-02128447}

Submitted on 14 May 2019

HAL is a multi-disciplinary open access archive for the deposit and dissemination of scientific research documents, whether they are published or not. The documents may come from teaching and research institutions in France or abroad, or from public or private research centers.
L'archive ouverte pluridisciplinaire HAL, est destinée au dépôt et à la diffusion de documents scientifiques de niveau recherche, publiés ou non, émanant des établissements d'enseignement et de recherche français ou étrangers, des laboratoires publics ou privés. 


\title{
On the occurrence of size effects in the calculation of thermal conductivity by first-principles molecular dynamics: the case of glassy $\mathrm{GeTe}_{4}$
}

\author{
Evelyne Martin ${ }^{\mathrm{a}}$, Pier Luca Palla ${ }^{\mathrm{a}}$, Fabrizio Cleri ${ }^{\mathrm{a}}$, Assil Bouzid ${ }^{\mathrm{b}}$, Guido Ori ${ }^{\mathrm{c}}$, Sébastien Le Roux ${ }^{\mathrm{c}}$, Mauro Boero ${ }^{\mathrm{c}}$, \\ Carlo Massobrio ${ }^{\mathrm{c}}$ \\ ${ }^{a}$ Univ. Lille, CNRS, Centrale Lille, ISEN, Univ. Valenciennes, UMR 8520 - IEMN, F-59000 Lille, France \\ ${ }^{b}$ Chaire de Simulation à l'Echelle Atomique (CSEA), Ecole Polytechnique Fédérale de Lausanne (EPFL), CH-1015 Lausanne, Switzerland. \\ ${ }^{c}$ Université de Strasbourg, CNRS, Institut de Physique et Chimie des Matériaux de Strasbourg, UMR 7504, F-67034 Strasbourg, France
}

\begin{abstract}
The thermal conductivity of a glass can be obtained by first-principles molecular dynamics provided we exploit a methodology that has been termed the approach-to-equilibrium molecular dynamics (AEMD) [1] [2, Chap. 8]. In the present work, we investigate the occurrence of size effects by comparing the thermal conductivity of two g-GeTe 4 atomic models of different sizes. This issue is far from being trivial since, in principle, size effects are not expected to occur in disordered systems beyond a few interatomic distances. For this reason, it is important to search unambiguous pieces of evidence substantiating this point. The first system of length $L=18 \AA$ contains 185 atoms. By duplicating it along one direction we form the second system, that contains 370 atoms and features a double length $L=36 \AA$ and an identical cross section. The thermal conductivity increases by a factor 3 from $L=18$ to $36 \AA$, thereby approaching the experimental value. Our investigation exemplifies the crucial role of the system size to take full advantage of the AEMD methodology and bring the calculated values in better agreement with experiments.
\end{abstract}

Keywords: Thermal Conductivity, Glass, First-Principles Molecular Dynamics

\section{Introduction}

Molecular dynamics (MD) simulations are widely used to obtain the thermal conductivity of bulk systems, nanostructures and interfaces $[3,1,4,5,6,7]$. In the literature, the forces between atoms are described by classical force fields with few exceptions. This is due to the need of extended MD trajectories to determine the thermal conductivity and obtain a fully converged autocorrelation function in the Green-Kubo approach [8], or to reach the steady state in the direct method [3]. However, it is highly desirable to go beyond the use of classical force fields, in particular in the case of glasses, since for disordered structures the use of quantitative models has found to be crucial $[9,10,11]$. This is specifically relevant for the class of chalcogenide glasses, for which empirical potentials often fail to quantitatively describe their structure and chemical interactions [12]. Recently [13], we have demonstrated that it is possible to determine the thermal conductivity of a glass, g-GeTe ${ }_{4}$,

Email address: evelyne.lampin@univ-lille.fr (Evelyne Martin)

Preprint submitted to Journal Name
20 from a density functional (DFT) based first-principles 21 MD (FPMD). The calculations have been performed by applying a methodology that we recently developed, the approach-to-equilibrium MD (AEMD) [1] [2, Chap. 8]. In this methodology, a transient regime is created and exploited to calculate the thermal conductivity in a way that proved to be faster and more effective than conventional approaches.

Past experience of application of the AEMD methodology provided evidence of the sensitivity to the system size of thermal conductivities obtained by AEMD. In particular, it has been shown that in crystalline $\mathrm{Si}(\mathrm{cSi})$, the thermal conductivity changes with the length of the system even up to $1.2 \mu \mathrm{m}$. This length dependence is intimately related [14] to the existence of long phonon free paths in cSi (larger than $10 \mu \mathrm{m}$ ) [15] [16]. In a disordered material like $\mathrm{g}-\mathrm{GeTe}_{4}$, the free paths of heat carriers are expected to be very short. At low temperature $(T \approx 130 \mathrm{~K})$, a mean free path (MFP) of $4-5 \AA$ has been measured by Zhang et al. [17]. However, it is known that the maximum heat carrier free paths can be considerably larger than the average value extracted from the thermal conductivity using the kinetic theory. 


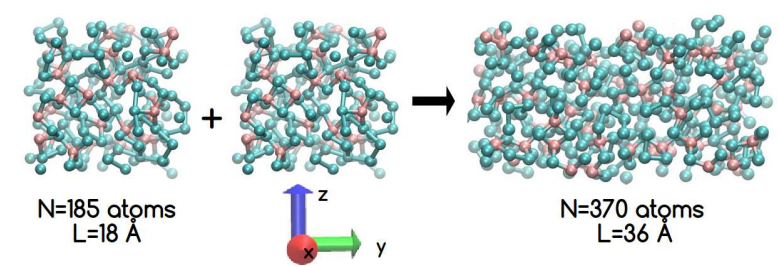

Figure 1: The atomic models containing respectively 185 and 370 atoms.

The atomic structure of the two systems is identical, 7 as evidenced by the comparison of the total pair correlation functions $g(r)$ (Fig. 2).

The details of the CPMD calculations are as follows. The Becke, Lee, Yang and Parr (BLYP) [19, 20] exchange-correlation functional is used, with a norm-conserving pseudo-potentials [21] describing the valence-core interaction. Long-range dispersion forces

\footnotetext{
${ }^{1}$ See http://www.cpmd.org, copyright 2000-2017 jointly by IBM 100 Corporation and by Max Planck Institute, Stuttgart.
}

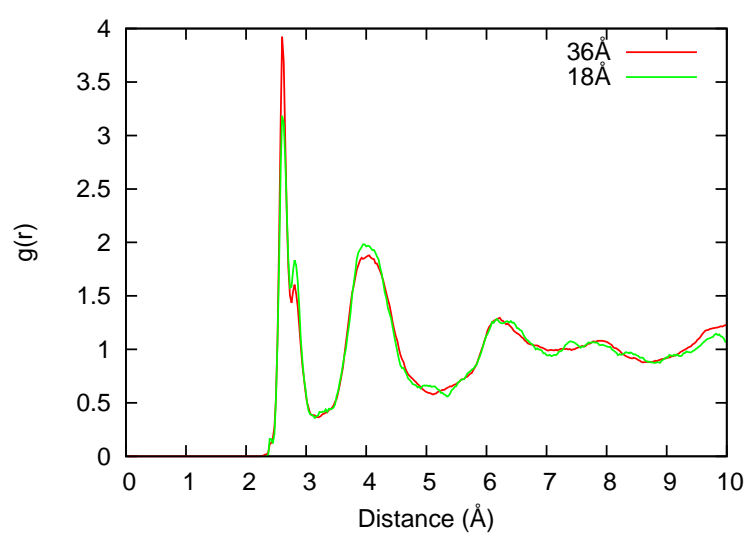

Figure 2: Pair correlation function $g(r)$ for the system of length $L=18 \AA$ containing 185 atoms and the system of length $L=36 \AA$ containing 370 atoms. The number of first neighbors as obtained from the integration of the first peak is equal to 3.8 in both cases.

are considered by following the formula by Grimme $[22,10]$. Thermostats are applied to the fictitious electronic degrees of freedom by following the Blöchl and Parrinello guidelines [23].

For each of the systems under study, the atomic structure is equilibrated during 4 to $30 \mathrm{ps}$ at the target temperature of $T_{t}=130 \mathrm{~K}$ since the MFPs are measured to be the lowest around this temperature [17]. The AEMD methodology is then applied to establish a thermal transient. The simulation box is divided into two sub-parts along the $y$-direction, these bound to be the cold and hot parts. To this aim, two distinct Nosé-Hoover [24, 25] thermostats are applied to the blocks, with a temperature difference $\Delta T_{0}$. Fig. 3 shows the time evolution of the temperature of the hot and the cold blocks in this phase 1 of AEMD for $\Delta T_{0}=200 \mathrm{~K}$. The temperature gap establishes in a few ps and it is stabilized by the application of the two thermostats along a time interval lasting 25 ps. Then, the thermostats are switched off to allow for the phase 2 of AEMD to begin. As a consequence, the temperature of the hot (cold) block decreases (increases). The equilibrium temperature of 130 $\mathrm{K}$ is reached faster for the smaller system (Fig. 3).

\section{Results}

The temperature difference during phase 2 of AEMD shown in Fig. 4 follows an exponential decay as exemplified by the linear variation on the semi-log graph. The decay time $\tau$ is extracted by a suitable fit.

We exploit the relationship between the decay time and thermal conductivity derived from the heat equation 


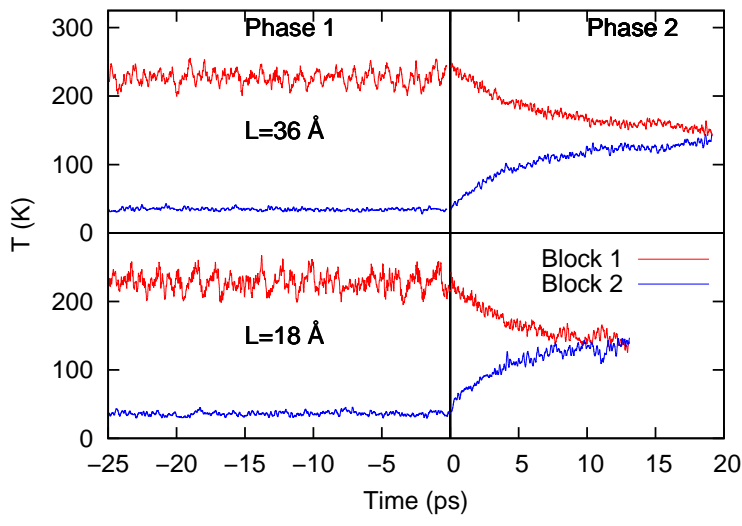

Figure 3: Time evolution of the hot block (red line) and cold block (blue line) during the two AEMD phases. Upper graph: $L=36 \AA$. Lower graph: $L=18 \AA$.

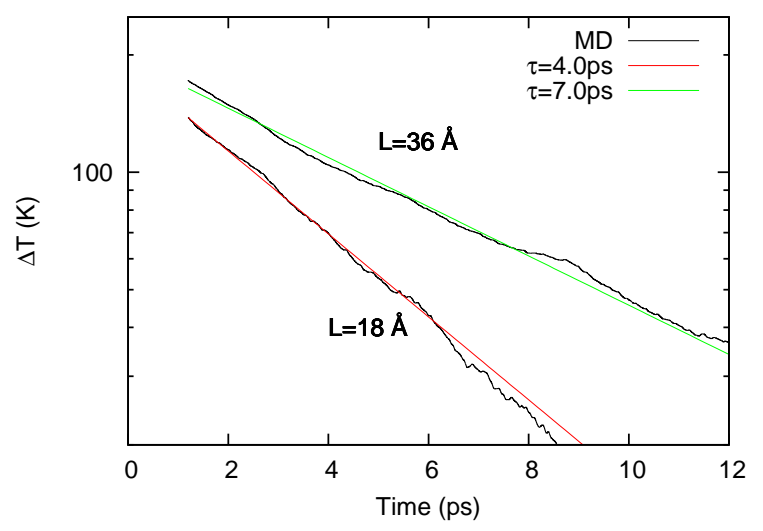

Figure 4: Time evolution of the temperature difference in phase 2 for the two systems and adjustment by a decaying exponential (the decay time is mentioned in label).

[1]:

$$
\kappa=\frac{L^{2}}{4 \pi^{2}} \frac{C_{V} \cdot \rho}{\tau}
$$

where $\rho=N / L^{3}$ is the number density. The specific heat $C_{V}=3 k_{\mathrm{B}}(0.78 \pm 0.02)$ is calculated from the energy 140 fluctuations in a $\{N V T\}$ run at $130 \mathrm{~K}$ [26].

The thermal conductivity is plotted as a function of 142 the system length in Fig. 5. For each size, the param- 143 eters of the simulation (like the initial temperature dif- 144 ference or the duration of phase 1) are varied in order to 145 ascertain their impact on the thermal conductivity. The 146 thermal conductivity varies without any clear cut depen- 147 dence on the different parameters. The variations are ${ }_{148}$ taken as the statistical error on $\kappa$. The calculations result ${ }_{149}$ in averaged thermal conductivity equal to $0.013 \pm 0.003 \quad 150$ $\mathrm{W} \mathrm{m}{ }^{-1} \mathrm{~K}^{-1}$ for $L=18 \AA$ and $0.044 \pm 0.001 \mathrm{~W} \mathrm{~m}^{-1} \mathrm{~K}^{-1}{ }_{151}$ for $L=36 \AA$. Therefore, the thermal conductivity features a length dependence, and increases by a factor of 3 from $L=18$ to $36 \AA$. We recall that the experimental value obtained using a parallel temperature conductance technique is $0.14 \mathrm{~W} \mathrm{~m}^{-1} \mathrm{~K}^{-1}$ [17]. This technique is reportedly leading to an overestimate by $20-25 \%$ of the thermal conductivity compared to a laser flash measurement [17]. This latest experimental framework is closer to our simulation methodology, and our experimental point of comparison is therefore a thermal conductivity of $\approx 0.1 \mathrm{~W} \mathrm{~m}^{-1} \mathrm{~K}^{-1}$.

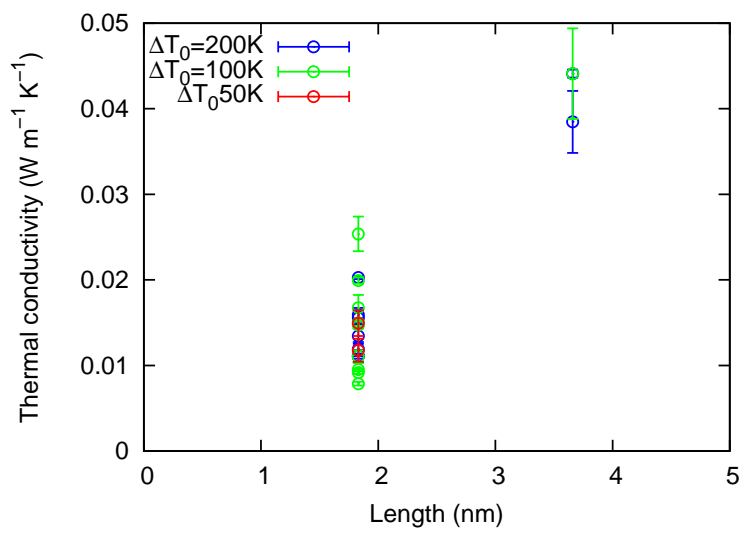

Figure 5: Thermal conductivity as a function of the length and for 3 values of the initial difference in temperature between hot and cold blocks $\left(\Delta T_{0}=200,100\right.$ or $\left.50 \mathrm{~K}\right)$ for atomic models containing respectively 185 and 370 atoms.

\section{Discussion}

There are several examples of length dependence of the thermal conductivity obtained using the AEMD methodology. In bulk silicon and germanium [1] [14], the thermal conductivity changes on the entire range of lengths studied, from tens of nanometers up to $1.2 \mu \mathrm{m}$. In $\alpha$-quartz [14], the thermal conductivity varies up to $\approx$ $100 \mathrm{~nm}$ before reaching a plateau. In silicon nanowires [5], the thermal conductivity always reaches a plateau only after a length that increases with the nanowire diameter. In silicon membranes [27], the same behavior is observed, with the plateau obtained at shorter length in presence of holes. All these cases were studied using classical force fields.

We have shown [14] that this behavior is due to the truncation at length $L$ of the distribution of phonon mean free paths. In crystals, phonons encounter scattering events that make their lifetime finite, or the distance 
they cover finite. The average mean free paths $\Lambda_{\mathrm{ph}}$ is 200 estimated from the kinetic theory following:

$$
\kappa=\frac{1}{3} C_{V} v \Lambda_{\mathrm{ph}}
$$

$v$ being the sound velocity. Nevertheless, phonon mean free paths can be distributed up to a maximum $\Lambda_{\max }$ considerably larger than $\Lambda_{\mathrm{ph}}$. For example in silicon, $\Lambda_{\mathrm{ph}}=$ $40 \mathrm{~nm}$ and $\Lambda_{\max }>10 \mu \mathrm{m}$.

In amorphous materials and glasses, heat carriers are not expected to cover long distances due to the disorder of the atomic structure that could be seen schematically as a distribution of defects beyond the first neighbor shell. Indeed, Zhang et al. [17] have estimated $\Lambda_{\mathrm{ph}}$ to 4-5 $\AA$ from their measurements of the thermal conductivity of $\mathrm{g}-\mathrm{GeTe}_{4}$. In amorphous silicon studied by classical force fields [14], the thermal conductivity does not exhibit any length dependence above $5 \mathrm{~nm}$.

The variation of thermal conductivity obtained in the ${ }_{214}$ present work, ranging from $L=18$ to $36 \AA$ could be due to the fact that $L$ is still smaller than the maxi- ${ }_{216}^{215}$ mum phonon mean free path $\Lambda_{\max }$. This conjecture can ${ }_{217}^{216}$ only be validated by looking for the saturation in the 218 calculated values of the thermal conductivity. This re- ${ }^{219}$ quires the calculation of the thermal conductivity on a ${ }_{221}^{220}$ third, even more extended system. It remains true that 222 the present calculations have the pleasing effect of ap- 223 proaching the AEMD value to the experimental coun- ${ }^{224}$ terpart. Also, it has to be observed that the size scaling ${ }_{226}^{225}$ of the CPMD method is prohibitive and by no means 227 linear. For this reason, it might be of interest to resort to ${ }^{228}$ alternative schemes (as the so-called second generation ${ }^{229}$ CPMD [28]) in order to speed up the achievement of the ${ }_{231}^{230}$ planned calculation for a third, larger system size.

\section{Conclusion}

The thermal conductivity of a glass, g-GeTe 4 has ${ }^{237}$ been calculated from first-principles molecular dynam- ${ }^{238}$ ics. In particular, the dependence on the system length ${ }_{240}^{239}$ has been the focus of the work, since the AEMD 241 methodology has shown such a dependence in many ${ }^{242}$ other materials. Although the disordered nature of $g_{-}{ }^{243}$ $\mathrm{GeTe}_{4}$ and an average phonon mean free paths mea- ${ }_{245}$ sured to 4-5 $\AA$ in the literature were in favor of an ab- 246 sence of length dependence, we have obtained an in- ${ }^{247}$ crease by a factor 3 of the thermal conductivity when ${ }_{249}^{248}$ the cell length along the heat path changes from 18 to 250 $36 \AA$. This amounts to a calculated value much closer 251 to experimental data. Therefore, it appears that such a ${ }^{252}$ length dependence has some physical origins that can ${ }_{254}^{253}$ be traced back to the existence of maximum mean free 255 paths larger than the system size. Further investigations for even larger sizes will be instrumental to demonstrate that the combination of FPMD with the AEMD approach is able to provide fully size-converged, reliable values for the thermal conductivity, at least in the case of a prototypical disordered system.

\section{Acknowledgements}

We acknowledge Clustphy2 of IEMN, Pôle HPC Equip@Medo of the University of Strasbourg, and GENCI (DARI N. x2016095071) for computer time allocations. We acknowledge financial support from the Agence Nationale de la Recherche (ANR) within the framework of the project SIRENA No. ANR-17-CE090039.

\section{References}

[1] E. Lampin, P. L. Palla, P. A. Francioso, F. Cleri, Thermal Conductivity from Approach-to-Equilibrium Molecular Dynamics, J. Appl. Phys. 114 (2013) 033525:1-5.

[2] K. Termentzidis, Nanostructured Semiconductors: Amorphisation and Thermal Properties, PanStanford, $1^{\text {st }}$ edition, 2017.

[3] S. R. Schelling, P. K. Phillpot, P. Keblinski, Comparison of Atomic-Level Simulation Methods for Computing Thermal Conductivity, Phys. Rev. B 65 (2002) 144306:1-12.

[4] S. G. Volz, G. Chen, Molecular Dynamics Simulation of Thermal Conductivity of Silicon Nanowires, Appl. Phys. Lett. 75 (1999) 2056-2058.

[5] H. Zaoui, P. L. Palla, F. Cleri, E. Lampin, Fourier-Like Conduction and Finite One-Dimensional Thermal Conductivity in Long Silicon Nanowires by Approach-to-Equilibrium Molecular Dynamics, Phys. Rev. B 94 (2017) 104308:1-7.

[6] P. K. Schelling, S. R. Phillpot, P. Keblinski, Kapitza Conductance and Phonon Scattering at Grain Boundaries by Simulation, J. Appl. Phys. 95 (2004) 6082-6091.

[7] E. Lampin, Q.-H. Nguyen, P. A. Francioso, F. Cleri, Thermal Boundary Resistance at Silicon-Silica Interfaces by Molecular Dynamics Simulations, Appl. Phys. Lett. 100 (2012) 131906.

[8] R. Zwanzig, Time-Correlation Functions and Transport Coefficients in Statistical Mechanics, Ann. Rev. Phys. Chem. 16 (1965) 67-102.

[9] C. Massobrio, A. Pasquarello, Structural Properties of Amorphous $\mathrm{GeSe}_{2}$, J. Phys.: Condensed Matter 19 (2007) 415111.

[10] A. Bouzid, C. Massobrio, M. Boero, G. Ori, K. Sykina, E. Furet, Role of the Van der Waals Interactions and Impact of the Exchange-Correlation Functional in Determining the Structure of Glassy GeTe ${ }_{4}$, Phys. Rev. B 92 (2015) 134208:1-10.

[11] A. Bouzid, S. Gabardi, C. Massobrio, M. Boero, M. Bernasconi, First-Principles Study of Amorphous $\mathrm{Ga}_{4} \mathrm{Sb}_{6} \mathrm{Te}_{3}$ Phase-Change Alloys, Phys. Rev. B 91 (2015) 184201.

[12] A. Bouzid, S. Le Roux, G. Ori, M. Boero, C. Massobrio, Origin of Structural Analogies and Differences Between the Atomic Structures of $\mathrm{GeSe}_{4}$ and $\mathrm{GeS}_{4}$ Glasses: a First Principles Study, J. Chem. Phys. 143 (2015) 034504.

[13] A. Bouzid, H. Zaoui, P. L. Palla, G. Ori, M. Boero, C. Massobrio, F. Cleri, E. Lampin, Thermal Conductivity of glassy $\mathrm{GeTe}_{4}$ by First-Principles Molecular Dynamics, Phys. Chem. Chem. Phys. 19 (2017) 9729-9732. 
[14] H. Zaoui, P. L. Palla, F. Cleri, E. Lampin, Length Dependence of Thermal Conductivity by Approach-to-Equilibrium Molecular Dynamics, Phys. Rev. B 94 (2016) 033525:1-5.

[15] K. T. Regner, D. P. Sellan, Z. Su, C. H. Amon, A. J. H. McGaughey, J. A. Malen, Broadband Phonon Mean Free Path Contributions to Thermal Conductivity Measured using Frequency Domain Thermoreflectance, Nature Comm. 4 (2013) 1640:1:7.

[16] A. S. Henry, G. Chen, Spectral Phonon Transport Properties of Silicon Based on Molecular Dynamics Simulations and Lattice Dynamics, J. Comput. Theo. Nanoscience 5 (2008) 141-152.

[17] S.-N. Zhang, J. He, T.-J. Zhu, X.-B. Zhao, T. M. Tritt, Thermal Conductivity and Specific Heat of Bulk Amorphous Chalcogenides $\mathrm{Ge}_{20} \mathrm{Te}_{80-x} \mathrm{Se}_{x}(x=0,1,2,8)$, J. Non-Crystal. Solids 355 (2009) 79-83.

[18] R. Car, M. Parrinello, Unified Approach for Molecular Dynamics and Density-Functional Theory, Phys. Rev. Lett. 55 (1985) 2471-2474.

[19] A. D. Becke, Density-Functional Exchange-Energy Approximation with Correct Asymptotic Behavior, Phys. Rev. A 38 (1988) 3098-3100.

[20] C. Lee, W. Yang, R. G. Parr, Development of the Colle-Salvetti Correlation-Energy Formula into a Functional of the Electron Density, Phys. Rev. B 37 (1988) 785-789.

[21] N. Troullier, J. L. Martins, Efficient Pseudopotentials for PlaneWave Calculations, Phys. Rev. B 43 (1991) 1993-2006.

[22] S. Grimme, Semiempirical GGA-Type Density Functional Constructed with a Long-Range Dispersion Correction, J. Comput. Chem. 27 (2006) 1787-1799.

[23] P. E. Blöchl, M. Parrinello, Adiabaticity in First-Principles Molecular Dynamics, Phys. Rev. B 45 (1992) 9413-9416.

[24] S. Nosé, A Molecular Dynamics Method for Simulations in the Canonical Ensemble, Mol. Phys. 52 (1984) 255-268.

[25] W. G. Hoover, Canonical Dynamics: Equilibrium Phase-Space Distributions, Phys. Rev. A 31 (1985) 1695-1697.

[26] M. E. Tuckerman, Statistical Mechanics: Theory and Molecular Simulation, Oxford University Press, 2010.

[27] H. Zaoui, P. L. Palla, S. Giordano, F. Cleri, M. Verdier, D. Lacroix, J.-F. Robillard, K. Termentzidis, E. Martin, FourierLike Conducion and Finite One-Dimensional Thermal Conductivity in Long Silicon Nanowires by Approach-to-Equilibrium Molecular Dynamics, Submitted to Int. J. Heat Mass Transfer (2018).

[28] T. D. Kühne, Second Generation Car-Parrinello Molecular Dynamics, Wiley Interdisciplinary Rev.: Computation. Mol. Sc. 4 (2014) 391-406. 